\title{
Identification of the electroelastic coupling from full multi-physical fields measured at
} the micrometre scale

Amiot, Fabien; Hild, F.; Kanoufi, F.; Roger, J. P.

Published in:

JOURNAL OF PHYSICS D APPLIED PHYSICS

Link to article, DOI:

$10.1088 / 0022-3727 / 40 / 11 / 009$

Publication date:

2007

Document Version

Publisher's PDF, also known as Version of record

Link back to DTU Orbit

Citation (APA):

Amiot, F., Hild, F., Kanoufi, F., \& Roger, J. P. (2007). Identification of the electroelastic coupling from full multiphysical fields measured at the micrometre scale. JOURNAL OF PHYSICS D APPLIED PHYSICS, 40(11), 3314-3325. https://doi.org/10.1088/0022-3727/40/11/009

\section{General rights}

Copyright and moral rights for the publications made accessible in the public portal are retained by the authors and/or other copyright owners and it is a condition of accessing publications that users recognise and abide by the legal requirements associated with these rights.

- Users may download and print one copy of any publication from the public portal for the purpose of private study or research.

- You may not further distribute the material or use it for any profit-making activity or commercial gain

- You may freely distribute the URL identifying the publication in the public portal 


\title{
Identification of the electroelastic coupling from full multi-physical fields measured at the micrometre scale
}

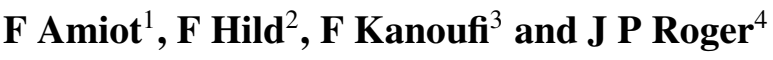 \\ ${ }^{1}$ MIC-DTU, Ørsted Plads, Bygning 345 Ø, Lyngby DK-2800, Denmark \\ ${ }^{2}$ LMT Cachan, ENS de Cachan / CNRS-UMR 8535 / Université Paris 6 , \\ 61 Avenue du Président Wilson, F-94235 Cachan Cedex, France \\ ${ }^{3}$ Laboratoire de Chimie Analytique, ESPCI / CNRS-UMR 7121, 10 rue Vauquelin, \\ Paris F-75005, France \\ ${ }^{4}$ Laboratoire d'Optique Physique, ESPCI / CNRS-UPR A0005 / Université Paris 6, \\ 10 rue Vauquelin, Paris F-75005, France \\ E-mail: fabien.amiot@mic.dtu.dk
}

Received 11 January 2007, in final form 4 April 2007

Published 18 May 2007

Online at stacks.iop.org/JPhysD/40/3314

\begin{abstract}
Metal coated microcantilevers are used as transducers of their electrochemical environment. Using the metallic layer of these cantilevers as a working electrode allows one to modify the electrochemical state of the cantilever surface. Since the mechanical behaviour of micrometre scale objects is significantly surface-driven, this environment modification induces bending of the cantilever. Using a full-field interferometric measurement set-up to monitor the objects then provides an optical phase map, which is found to originate from both electrochemical and mechanical effects. The scaling of the electrochemically-induced phase with respect to the surface charge density is modelled according to Gouy-Chapman-Stern theory, whereas the relationship between the mechanical effect and the surface charge density is analysed. An identification technique is described to determine a modelling of the electroelastic coupling and to identify the spatial charge density distribution from full-field phase measurements. Minimizing the least-squares gap between the measured phase and a statically admissible phase field, the mechanical effect is found to be charge-driven. The charge density field is also found to be singular on the cantilever edge, and the shear stress versus charge density is found to be non-linear.
\end{abstract}

(Some figures in this article are in colour only in the electronic version)

\section{Introduction}

The increasing interest in microelectromechanical systems (MEMS) has raised several issues concerning several specific mechanical phenomena. Decreasing the size of mechanical objects down to the $1-100 \mu \mathrm{m}$ range significantly enhances the surface-driven aspect of the mechanical behaviour, so that these objects are used in a wide range of sensing applications [1]. The present paper intends to focus on the coupling between the electrochemical and the mechanical effects, which involves small elastic strains, thus requiring the measurement of vanishingly small strains [2]. This was first achieved on macroscopic samples by several optical methods such as Köster interferometer [3], which was used with a gauge length in the $10 \mathrm{~mm}$ range. Electrochemical effects have also been investigated through the mechanical loading they induce on microcantilevers. An investigation of the double-layer region [4] (i.e. no electrochemical reaction) as well as adsorption reactions [5-7] and electrodeposition [8] have been carried out using the standard optical lever 
technique [1]. Using this set-up allows one to demonstrate the phenomenon, but it is not suited to describing the involved mechanical effect. The development of in situ ellipsometric measurements also allows for the development of comprehensive models describing the optical behaviour of noble metal working electrodes in the double-layer regime $[9,10]$. These models, based on the Gouy-Chapman-Stern theory, have been successfully used to monitor the anion adsorption in the double-layer regime [11], thus providing a description of the electrochemical state of the interface. The electroelastic coupling has then been monitored, combining the optical lever technique with ellipsometric measurements on a cantilever [12].

However, if the existence of this surface coupling phenomena has been demonstrated, the modelling of the connection between the electrochemical state of an interface and the induced deformation remains an open question [2]. Since one of the main difficulty is to ensure a uniform and well-defined loading at the micrometre scale, it is thought that increasing the experimental information amount may lead to a significant modelling improvement, provided the redundancy of the measured quantity is sufficient. This statement is at the origin of the development of identification techniques based on full-field measurements in solid mechanics.

A first class of identification techniques allows for the identification of elastic properties using redundant or full-field kinematic data in the typical case where the loading is applied in a well-defined manner. A first group, initiated in [13], is derived from the constitutive equation error [14] and has been applied to both dynamic model updating [15] and elastic property or damage field identification [16]. It was also used to get elastic properties by analysing a heterogeneous test [17]. Based on equilibrium conditions, the virtual fields method has been used to identify homogeneous elastic properties of composites $[18,19]$. The reciprocity gap [20] is a specific technique needing both kinematic and static quantities at the same location of the body boundary [21]. Dealing with experiments where the mechanical loading has to be identified represents another class of identification problems.

Starting from the equilibrium gap method [22], one may consider only kinematic measurements and add some unknowns (describing the loading) in the identification problem. This (inverse) problem is solved, provided that the mechanical behaviour remains elastic [23]. Dealing with multi-physical (i.e. coupled) phenomena, an alternative solution consists of enriching the experimental basis with full-field measurements of any complementary information describing the phenomenon under scrutiny. The case where these two fields are combined in a single measured field is hereby considered, thus requiring no additional measurement but a decoupling procedure. The aim of the present paper is to propose the use of phase-modulated interferometric measurements and an electrochemical workstation, presented in section 2, to have access to the change in a full phase field of the observed object, originating from both the electrochemical state of the interface and the mechanical effect on the observed cantilever. The way these two phenomena operate is described and modelled in section 3. The proposed decomposition allows one to distinguish the electrochemically and mechanically induced optical phase and thus to derive simultaneously a description of the charge density field and the electroelastic coupling through a dedicated identification procedure (section 4).

\section{Experimental set-up}

\subsection{Nomarski imaging set-up}

The interferential microscopy imaging set-up used herein is shown in figure 1. A light-emitting diode (LED, $\lambda=760 \mathrm{~nm}$ ) illuminates a polarization beam-splitter. The beam reflected by the beam-splitter is polarized at $45^{\circ}$ of the axes of a photoelastic polarization modulator. The Wollaston prism, whose axes are parallel to those of the modulator, splits the beam into two orthogonally polarized beams at a small angle between each other. These beams are focused upon the sample by an immersion objective lens ( $18 \mathrm{~mm}$ focal length, NA $=0.3$ ). After reflection and recombination by the Wollaston prism, the beam goes through the polarization modulator and the polarization beam-splitter. The transmitted beam is finally focused on a CCD array (Dalsa-CA-D1, $256 \times 256$ pixels, 8 bits). The polarization beam-splitter behaves as crossed linear polarizers mounted at $45^{\circ}$ of the axes of the Wollaston prism and of the polarization modulator.

The interference pattern is obtained as the difference of two topographies of the surface, shifted by the Wollaston prism by a distance $d$. The Wollaston shear sdirection is chosen to be parallel to the cantilever axis, denoted by $\mathbf{x}$, so that the topographies involved in the interference pattern are views of the cantilever shifted along the direction of its larger dimension

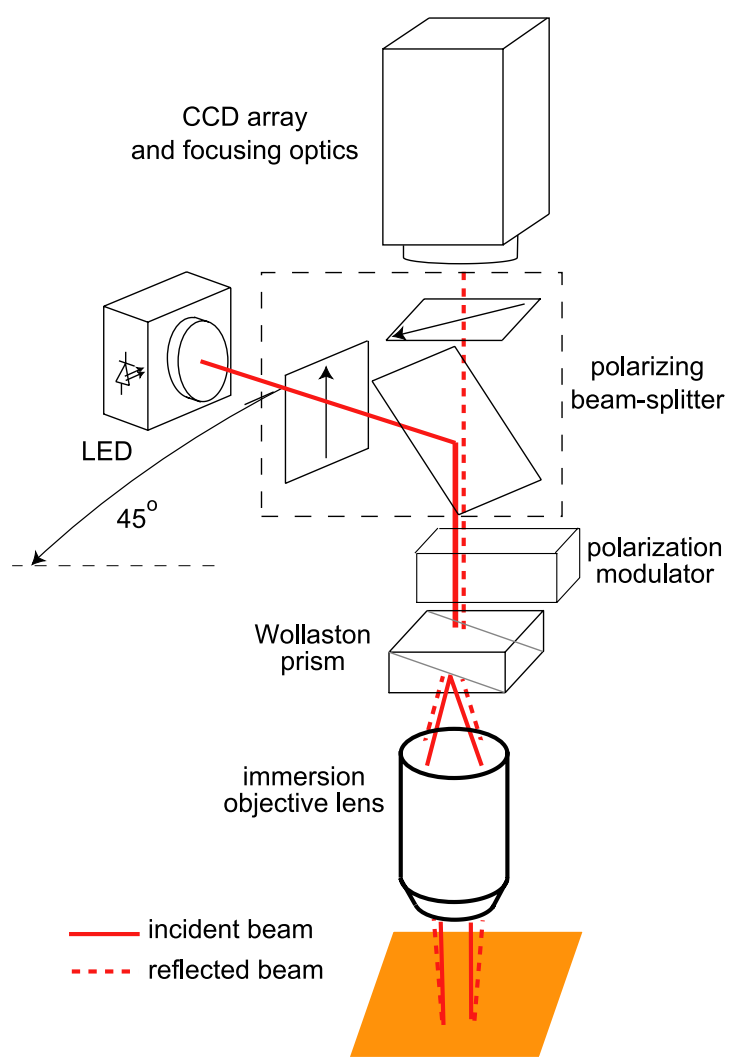

Figure 1. Schematic view of a Nomarski shear-interferometer with phase modulation. 


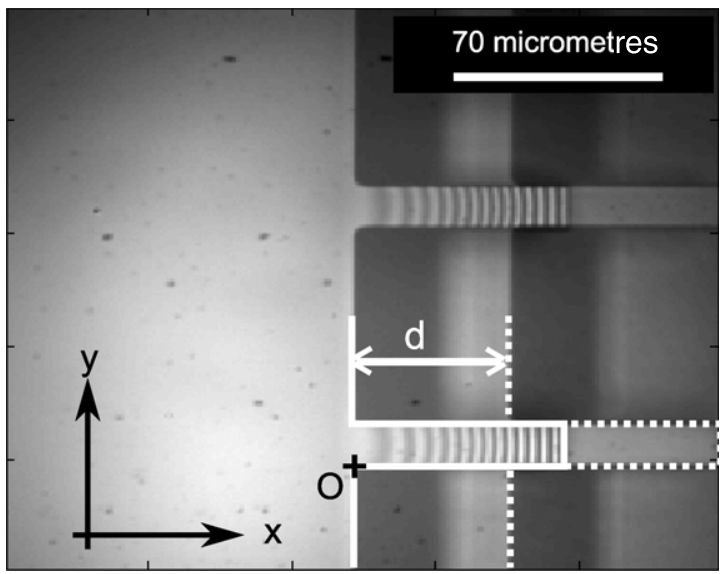

Figure 2. A typical interference pattern. The shear direction is parallel to the cantilever direction, and the shear $d$ is almost equal to the cantilever length. The axis system is shifted for the sake of clarity and the origin is set at point $\mathrm{O}$.

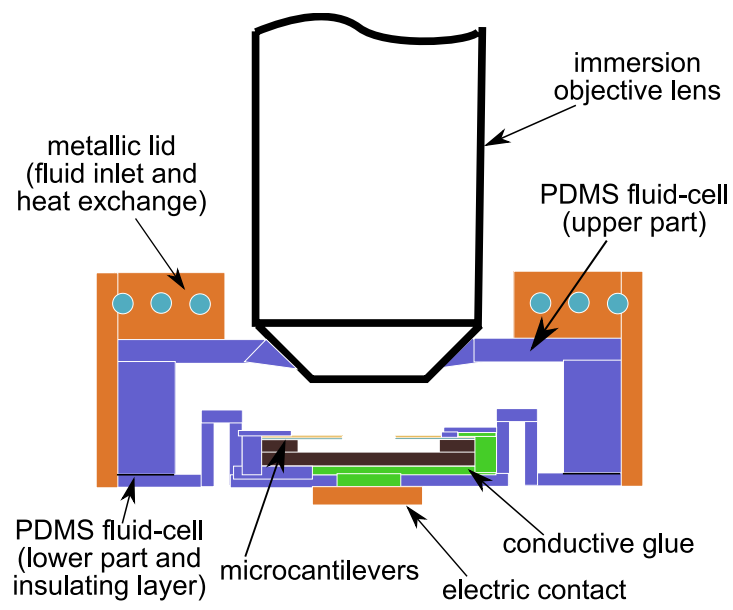

Figure 3. Schematic view of the used fluid cell.

(see figure 2). The distance $d$ is chosen to be almost similar to the cantilever length. The pixel size in the object plane is equal to $0.9 \mu \mathrm{m}$. The optical phase map is then retrieved using four integrating buckets and arises from both the sample topography (i.e. height and slope fields) and the reflection coefficient field of the interface [24].

\subsection{Electrochemical procedure}

2.2.1. Sample preparation. Figure 3 shows a schematic view of the used fluid cell. It is mainly composed of two polymer parts (polydimethylsiloxane (PDMS)). The lower part is used to hold the sample, which is glued on a copper piece thanks to a conducting epoxy resin. The temperature of this copper piece is controlled by Peltier effect modules and a temperature sensor. A clear aperture is made in the upper part and closed by the immersion objective. The mechanical contact between the objective and the upper PDMS part ensures the fluid cell to be watertight. Another copper part, whose temperature is also controlled by Peltier modules, embraces this upper polymer part. The temperature was set to $24.7^{\circ} \mathrm{C}$ during the experiment. Fluid inlet and outlet allow for a simple modification of the content of the fluid cell.

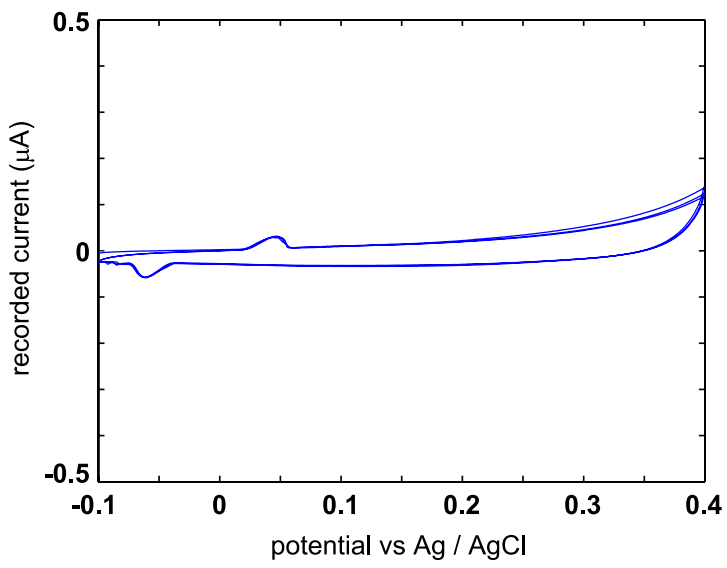

Figure 4. Cyclic voltammetry of the microcantilevers array in a $10^{-2} \mathrm{M} \mathrm{KCl}$ solution. Number of cycles: 3 , scan rate: $2 \mathrm{mV} \mathrm{s}^{-1}$, starting potential: $-0.1 \mathrm{~V}$ versus $\mathrm{Ag} / \mathrm{AgCl}$.

The fluid cell presented in figure 3 is filled with an electrolytic solution, and a gold-coated silica array of 18 cells of 6 micro-objects is sealed in it. The objects under consideration are microcantilevers $\left(70 \times 20 \times 0.84 \mu \mathrm{m}^{3}\right)$, which are made of silica (thickness: $770 \mathrm{~nm}$ ) obtained by thermal oxidation of a silicon wafer. The cantilevers are defined by patterning and isotropic etching of the silica (in HF buffer) and released by $\mathrm{KOH}$ etching. A titanium (thickness: $20 \mathrm{~nm}$ ) and a gold (thickness: $50 \mathrm{~nm}$ ) layer are then evaporated onto the sample. A conducting glue is used to ensure the electrical connection to the gold layer. This microcantilevers cell is used as the working electrode. All the connections are insulated with a thin PDMS layer, to ensure that only the gold surface of the working electrode is in contact with the solution. This insulation step was performed outside the cleanroom, by pipetting some PDMS around the sample and avoiding physical contact between PDMS and the cantilevers. There is then a total area of almost $1 \mathrm{~cm}^{2}$ in constant contact with the electrolytic solution. The counter-electrode is a $250 \mu \mathrm{m}$ diameter and $15 \mathrm{~cm}$ long platinum wire plunging in the fluid cell. The $\mathrm{Ag} / \mathrm{AgCl}$ reference electrode is made of a silver wire $(250 \mu \mathrm{m}$ in diameter) onto which a silver chloride layer has been electrodeposited by oxidation in a $1 \mathrm{M}$ $\mathrm{KCl}$ solution. The three electrodes are then connected to a CHI 660 A electrochemical workstation (IJCambria, England). After bubbling nitrogen, the fluid cell is then filled with a $\mathrm{KCl}$ solution $\left(10^{-2} \mathrm{M}\right)$, prepared from milliQ water. The gold surface is then cleaned electrochemically by performing successive cyclic voltammograms between 0 and $0.8 \mathrm{~V}$ at $10 \mathrm{mV} \mathrm{s}^{-1}$. Gold is then successively oxidized and reduced, until a stable voltammogram (i.e. after a few cycles) is obtained, thus ensuring a clean and reproducible metallic surface. The fluid cell is then purged and filled again with a $10^{-2} \mathrm{M} \mathrm{KCl}$ deoxygenated solution.

2.2.2. Electrochemical response of the microcantilevers array. Figure 4 shows the cyclic voltammetry at the microcantilevers array. The electrode potential was swept linearly at a scan rate of $2 \mathrm{mV} \mathrm{s}^{-1}$, during three cycles, between -0.1 and $0.4 \mathrm{~V}$ versus $\mathrm{Ag} / \mathrm{AgCl}$. The scan rate was chosen to ensure an almost constant state during an imaging step (about $1 \mathrm{~s}$ ), 
and the potential is prescribed with a $0.1 \mathrm{mV}$ resolution. The potential window was chosen so that the electrode processes are not perturbed by any substance or electrode material electrochemical transformation. For potentials less than $-0.1 \mathrm{~V}$ versus $\mathrm{Ag} / \mathrm{AgCl}$, oxygen reduction may be observed while a peak, presumably related to Au oxidation, is observed for potentials greater than $0.5 \mathrm{~V}$. The reversible system observed at $0.05 \mathrm{~V}$ (oxidation) and $-0.05 \mathrm{~V}$ (reduction) is attributed to the Ti underlayer oxidation. The shape of these peaks (namely, symmetric peaks 'added' to a base line) is typical for surface, diffusionless, phenomena such as surface passivation. The related electrochemical process is therefore believed not to interfere with the double-layer charging on the gold layer. The shape of the voltammogram, in the chosen potential region, is characteristic of a double layer charging process. It is worth noting that voltammograms obtained at different scan rates are found to change proportionally to the scan rate (except around the reversible system), thereby proving the non-Faradaic nature of the measured current. The numerical integration of the cyclic voltammogram is obtained by using all the three cycles sampled with a $1 \mathrm{mV}$ resolution, thus providing the variation of the charge carried by the electrode with the potential.

\section{Phenomena at the origin of the measured optical phase}

During the three potential cycles, a phase map is recorded every $0.05 \mathrm{~V}$ step, thus providing a set of 61 phase maps, that is a $15 \times 111 \times 61$ phase measurements stack covering the cantilever through the whole experiment. The aim of this section is then to express the phase change as a function of both the deformation of the cantilever and the electrochemical modification of the interface.

\subsection{Decomposition in electrochemically induced and mechanically induced optical phase}

The interface composition is modified when subjected to a potential change. This property has been used to predict the change of the ellipsometric parameters with the surface charge in these surfaces $[9,10]$. Paik et al [11] have shown that it is necessary to take into account at least two phenomena if one wants to compute the ellipsometric parameters change in an electrode/electrolyte interface:

- the local change in the refractive index of the solution due to a local concentration shift;

- the complex refractive index change in the metal induced by the free electrons density change at the surface.

If the electrode potential is modified to impose a metal surface charge $\sigma_{\mathrm{m}}$, then the electrolyte side of the interface yields a surface charge density $\sigma_{\mathrm{d}}$ in a diffuse layer, and specific adsorption of anions (respectively, cations) may occur, leading to a surface charge $\sigma_{-}$(respectively, $\left.\sigma_{+}\right)$. Electrical equilibrium of the interface requires

$$
\sigma_{\mathrm{m}}+\sigma_{\mathrm{d}}+\sigma_{-}+\sigma_{+}=0
$$

The Gouy-Chapman theory [25-27] may be used to describe the interface between a monovalent electrolyte solution whose bulk concentration is $N_{0}$ (i.e. number of charges per unit volume) and a metallic electrode, assuming the latter to be planar. The diffuse layer charge per unit electrode surface $\sigma_{\mathrm{d}}$ reads as

$$
\sigma_{\mathrm{d}}=-\sqrt{8 N_{0} \epsilon_{\mathrm{w}} k T} \sinh \left\{\frac{e\left(\Phi_{\mathrm{m}}-\Phi_{\infty}\right)}{2 k T}\right\},
$$

where $\epsilon_{\mathrm{w}}$ is the dielectric constant for water, $k$ the Boltzmann constant, $T$ the absolute temperature, $e$ the electron charge, $\Phi_{\mathrm{m}}$ the electrode (metal) potential and $\Phi_{\infty}$ the remote potential. The concentration distributions for cations $N_{+}$and anions $N_{-}$ read as

$$
N_{ \pm}=N_{0}\left(\frac{1 \pm \gamma \exp \left(-\frac{x}{\lambda_{\mathrm{D}}}\right)}{1 \mp \gamma \exp \left(-\frac{x}{\lambda_{\mathrm{D}}}\right)}\right)^{2},
$$

where the parameter $\gamma$ is defined by

$$
\gamma=\tanh \left\{\frac{-e\left(\Phi_{\mathrm{m}}-\Phi_{\infty}\right)}{4 k T}\right\}
$$

and $\lambda_{\mathrm{D}}$ is the Debye length given by

$$
\lambda_{\mathrm{D}}^{2}=\frac{\epsilon_{\mathrm{w}} k T}{8 \pi e^{2} N_{0}} .
$$

From equation (3), it is shown that only a thin layer close to the surface, whose thickness scales as $\lambda_{\mathrm{D}}$, is significantly modified when charging the electrode. The concentration shift induced in this layer modifies locally the refractive index of the solution, modifying the complex reflection coefficient of the interface, and therefore generating a non-mechanically induced optical phase change. For the present system, $\lambda_{\mathrm{D}} \simeq$ $3 \mathrm{~nm}$. Consequently, the total measured optical phase change $\phi$ is decomposed into a mechanically induced optical phase $\phi_{\mathrm{mec}}$ and an electrochemically induced optical phase $\phi_{\mathrm{ec}}$

$$
\phi=\phi_{\mathrm{mec}}+\phi_{\mathrm{ec}}
$$

The combination is assumed to be additive, as long as one term significantly dominates the other one:

- the 'almost mechanical' case, where the displacement is large compared with $\lambda_{\mathrm{D}}$,

- the 'almost electrochemical' case, where $\lambda_{\mathrm{D}}$ is large compared with the displacement.

The following two subsections are intended to relate $\phi_{\mathrm{mec}}$ and $\phi_{\text {ec }}$ terms to the surface charge density $\sigma_{\mathrm{d}}$.

\subsection{Electrochemically induced optical phase}

One proposes to compute the reflection coefficient of a charged interface for a focused beam in the same manner as Stedman $[9,10]$ and Paik et al [11] derived the ellipsometric parameters for a plane wave impinging on a metal-electrolyte interface. Let us derive refractive index changes of the solution and of the metal for the present case and use them to describe the link between $\phi_{\mathrm{ec}}$ and the electrochemical state of the interface through the complex reflection coefficient change of the surface illuminated with a focused monochromatic beam. 
3.2.1. Refractive index of the solution. The surface excess concentrations are deduced from the density profiles

$$
\Gamma_{ \pm}=\int_{0}^{\infty}\left(N_{ \pm}-N_{0}\right) \mathrm{d} z=N_{0} \frac{ \pm 4 \gamma \lambda_{\mathrm{D}}}{1 \mp \gamma} .
$$

As previously discussed [9], one chooses to represent the diffuse layer by a finite one whose thickness is $2 \lambda_{\mathrm{D}}$. One also considers that the liquid side of the interface is charged by the diffuse layer, as well as by adsorbed ions located at the inner Helmholtz plane. The equivalent densities $\tilde{N}_{+}$and $\tilde{N}_{-}$ are thus defined to ensure the same surface excesses

$$
\begin{aligned}
& \tilde{N}_{+}=N_{0}+\frac{\Gamma_{+}+\sigma_{+}}{2 \lambda_{\mathrm{D}}}=N_{0} \frac{1+\gamma}{1-\gamma}+\frac{\sigma_{+}}{2 \lambda_{\mathrm{D}}}, \\
& \tilde{N}_{-}=N_{0}+\frac{\Gamma_{-}+\sigma_{-}}{2 \lambda_{\mathrm{D}}}=N_{0} \frac{1-\gamma}{1+\gamma}+\frac{\sigma_{-}}{2 \lambda_{\mathrm{D}}} .
\end{aligned}
$$

The refractive index $n_{\mathrm{e}}$ of the electrolyte layer is then obtained from the Lorentz-Lorenz formula

$$
n_{\mathrm{e}}\left(\Phi_{\mathrm{m}}-\Phi_{\infty}, \sigma_{+}, \sigma_{-}\right)=\sqrt{\frac{\bar{M}+2 \bar{\rho} \sum_{i} x_{i} R_{i}}{\bar{M}-\bar{\rho} \sum_{i} x_{i} R_{i}}},
$$

where $\bar{M}$ is the mean molar mass of the film and $\bar{\rho}$ its mean mass density. The latter requires the molar volume of the components of the film, which are taken from [28]. The molar fractions $x_{i}$ of the different elements composing the film read as

$$
x_{i}=\frac{\tilde{N}_{i}}{\tilde{N}_{\mathrm{H}_{2} \mathrm{O}}+\tilde{N}_{+}+\tilde{N}_{-}}
$$

for $i=\left\{\mathrm{H}_{2} \mathrm{O},+,-\right\}$. The molar refractivities $R_{i}$ of the components of the film are taken from [29].

3.2.2. Refractive index of the metal. According to Drude's model, and neglecting the contribution of the bound electrons, the complex refractive index $\hat{n}_{\mathrm{m}}$ of the metal is obtained as

$$
\hat{n}_{\mathrm{m}}^{2}=1-\frac{4 \pi n_{\mathrm{el}} e^{2}}{m} \frac{1}{\omega(\omega-\mathrm{i} \beta)},
$$

where $\beta$ is Drude's damping parameter, $m$ the electron mass, $\omega$ the frequency of the impinging wave and $n_{\mathrm{el}}$ the volume charge density. Decomposing $\hat{n}_{\mathrm{m}}$ into real and imaginary parts

$$
\hat{n}_{\mathrm{m}}=n_{\mathrm{m}}(1+\mathrm{i} \kappa)
$$

and considering both real and imaginary parts of equation (12) yields

$$
\begin{gathered}
n_{\mathrm{m}}^{2}\left(1-\kappa^{2}\right)=1-s n_{\mathrm{el}}, \\
2 n_{\mathrm{m}}^{2} \kappa=-s \frac{\beta}{\omega} n_{\mathrm{el}},
\end{gathered}
$$

where $s$ is defined by

$$
s=\frac{4 \pi e^{2}}{m} \frac{1}{\omega^{2}+\beta^{2}} .
$$

Solving equation (14) for the ratio $\beta / \omega$ yields

$$
\frac{\beta}{\omega}=-\frac{2 n_{\mathrm{m}}^{2} \kappa}{1-n_{\mathrm{m}}^{2}\left(1-\kappa^{2}\right)} .
$$

The ratio $\beta / \omega$ is deduced from ellipsometric measurements. At zero charge, one measures

$$
\begin{gathered}
n_{\mathrm{m}}=0.33, \\
\kappa=-13.12
\end{gathered}
$$

for a wavelength of $760 \mathrm{~nm}$. Setting (16) into its definition, $s$ is rewritten as

$$
s=\frac{4 \pi e^{2}}{m \omega^{2}} \frac{\left(1-n_{\mathrm{m}}^{2}\left(1-\kappa^{2}\right)\right)^{2}}{1-2 n_{\mathrm{m}}^{2}\left(1-\kappa^{2}\right)+n_{\mathrm{m}}^{4}\left(1+\kappa^{2}\right)^{2}} .
$$

If the surface charge density $\sigma_{\mathrm{m}}$ is changed to $\sigma_{\mathrm{m}}+\mathrm{d} \sigma_{\mathrm{m}}$, the charge density change at the surface reads as

$$
\mathrm{d} n_{\mathrm{el}}=\frac{\mathrm{d} \sigma_{\mathrm{m}}}{e t}
$$

where $t$ is the 'thickness' of the metal layer whose charge is modified. One arbitrarily sets $t=1 \mathrm{~nm}$, as in [11]. The new refractive index of the metal layer is described by $n_{\mathrm{m}}^{\prime}$ and $\kappa^{\prime}$ satisfying

$$
\begin{aligned}
n_{\mathrm{m}}^{\prime 2}\left(1-\kappa^{\prime 2}\right) & =n_{\mathrm{m}}^{2}\left(1-\kappa^{2}\right)-s \times \mathrm{d} n_{\mathrm{el}}, \\
2 n_{\mathrm{m}}^{\prime 2} \kappa^{\prime} & =2 n_{\mathrm{m}}^{2} \kappa-s \frac{\beta}{\omega} \mathrm{d} n_{\mathrm{el}} .
\end{aligned}
$$

$n_{\mathrm{m}}^{\prime}$ is thus found by solving

$$
\begin{aligned}
4 n_{\mathrm{m}}^{\prime 4} & +n_{\mathrm{m}}^{\prime 2}\left[4 n_{\mathrm{m}}^{2}\left(\kappa^{2}-1\right)+4 s \mathrm{~d} n_{\mathrm{el}}\right] \\
& -4 n_{\mathrm{m}}^{2}\left(n_{\mathrm{m}}^{2} \kappa^{2}-\kappa s \frac{\beta}{\omega} \mathrm{d} n_{\mathrm{el}}\right)-\left(s \frac{\beta}{\omega} \mathrm{d} n_{\mathrm{el}}\right)^{2}=0
\end{aligned}
$$

and $\kappa^{\prime}$ is deduced from

$$
\kappa^{\prime}=\frac{2 n_{\mathrm{m}}^{2} \kappa-s \frac{\beta}{\omega} \mathrm{d} n_{\mathrm{el}}}{2 n_{\mathrm{m}}^{\prime 2}} .
$$

3.2.3. Reflection coefficient of the surface. The complex reflection coefficients $r_{\mathrm{TE}}$ and $r_{\mathrm{TM}}$ for TE and TM polarized light are then deduced using the Fresnel formula [30] as functions of the surface charge density in the metal $\sigma_{\mathrm{m}}$ and the charge densities for adsorbed anions $\sigma_{-}$and cations $\sigma_{+}$, modelling the interface with two homogeneous layers whose optical properties are defined as described above. The electrochemical contribution to the optical phase field measured with the Nomarski shear-interferometer is then described considering TE and TM polarized beams separately, for a given incidence angle $\theta$. For example,

$$
\phi_{\mathrm{ec}}(x, y, \theta)=\phi_{\mathrm{TM}}(x, y, \theta)-\phi_{\mathrm{TE}}(x-d, y, \theta) .
$$

As the image is formed by using the whole objective pupil, the phase change measured after reflection on the sample does not depend on the initial polarization [31], so that the fields $\phi_{\mathrm{TM}}$ and $\phi_{\mathrm{TE}}$ are equal. As a consequence, $\phi_{\mathrm{ec}}(x, y, \theta)$ is a measure of the heterogeneous part of the reflection phase change field

$\phi_{\mathrm{ec}}(x, y, \theta)=\phi_{\mathrm{TE}, \mathrm{TM}}(x, y, \theta)-\phi_{\mathrm{TE}, \mathrm{TM}}(x-d, y, \theta)$.

The exact relationship for the fully illuminated pupil may be difficult to predict accurately since it depends on the numerical aperture and the apodization function of the 


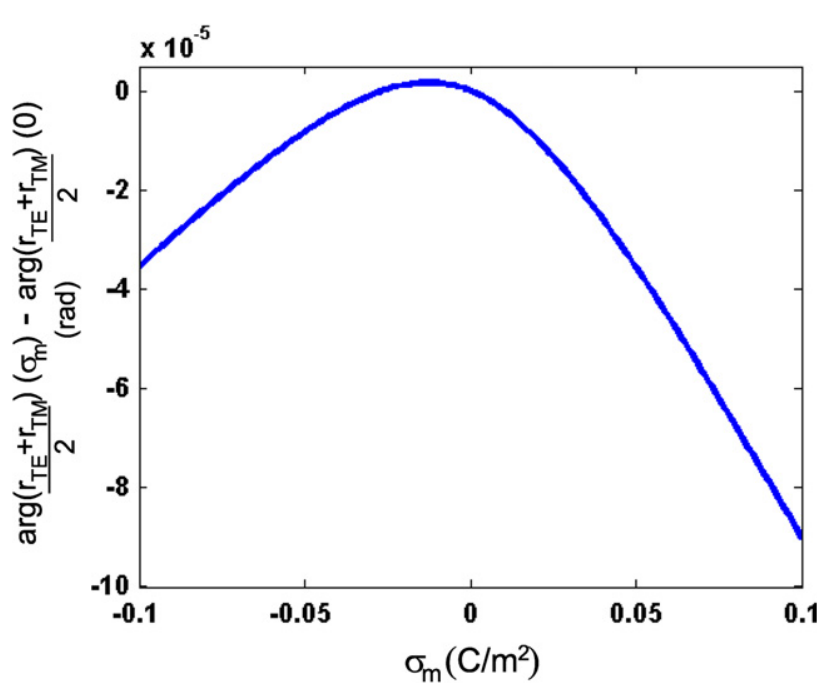

Figure 5. Change in the phase of the base complex reflection coefficient $\frac{r_{\mathrm{TE}}+r_{\mathrm{TM}}}{2}$ of a $\mathrm{Au}\left(\begin{array}{lll}1 & 1 & 1\end{array}\right) / \mathrm{KCl}$ interface as a function of the surface charge for $\theta_{\mathrm{e}}=0.3 \mathrm{rad}$.

objective. Therefore, one will consider in the following the function $\phi_{\mathrm{TE}, \mathrm{TM}}\left(x, y, \theta_{\mathrm{e}}\right)=h\left(\sigma_{\mathrm{m}}(x, y), \theta_{\mathrm{e}}\right)$ of the metal surface charge density $\sigma_{\mathrm{m}}(x, y)$ and of an apparent incidence angle $\theta_{\mathrm{e}}[32]$ :

$\phi_{\mathrm{TE}, \mathrm{TM}}\left(x, y, \theta_{\mathrm{e}}\right)=\arg \left(\frac{r_{\mathrm{TE}}\left(x, y, \theta_{\mathrm{e}}\right)+r_{\mathrm{TM}}\left(x, y, \theta_{\mathrm{e}}\right)}{2}\right)$

$=h\left(\sigma_{\mathrm{m}}(x, y), \theta_{\mathrm{e}}\right)$.

Figure 5 shows the change in the phase of the base complex reflection coefficient $\left(r_{\mathrm{TE}}+r_{\mathrm{TM}}\right) / 2$ of the considered interface as a function of the electrode surface charge, assuming no specific adsorption (i.e. $\sigma_{-}=\sigma_{+}=0$ ), for $\theta_{\mathrm{e}}=0.3 \mathrm{rad}$. It is worth noting that the described results are consistent with previously published ellipsometric parameters [11]. Moreover, as outlined in [11], the obtained phase function does not significantly depend on the arbitrarily chosen 'thickness' of the affected metal layer.

\subsection{Mechanically induced optical phase}

3.3.1. From Gouy-Chapman theory to a surface shear-stress field. Considering that the electrochemical free enthalpy $\mathcal{G}_{S}$ is driven by the interactions between the ions in solution and the charges on the electrode, the total free enthalpy variation of a small surface $S_{0}$ subjected to a small surface change $\delta S$ is written as

$$
\delta \mathcal{G}=\delta \mathcal{G}_{\mathrm{e}}+\delta \mathcal{G}_{S}=\delta \mathcal{G}_{\mathrm{e}}(\delta S)+\delta S \frac{\sigma_{\mathrm{m}}^{2}}{\epsilon_{\mathrm{w}}},
$$

where the strain energy term $\delta \mathcal{G}_{\mathrm{e}}(\delta S)$ depends on the mechanical behaviour of the cantilever. Let us denote by $u(x)$ the in-plane displacement field of the cantilever surface. The surface modification then reads as

$$
\delta S(x)=b u(x),
$$

where $b$ is the cantilever width. Replacing it in equation (25) leads to

$$
\delta \mathcal{G}(x)=\delta \mathcal{G}_{\mathrm{e}}(x)+\delta \mathcal{G}_{S}(x)=\delta \mathcal{G}_{\mathrm{e}}(x)+b u(x) \frac{\sigma_{\mathrm{m}}^{2}}{\epsilon_{\mathrm{w}}},
$$

so that the electrochemical term appears as an external force contribution, similar to the work of a shear stress $\tau(x)$ in the in-plane displacement $u(x)$

$$
\delta \mathcal{G}(x)=\delta \mathcal{G}_{\mathrm{e}}(x)+b u(x) \frac{\sigma_{\mathrm{m}}^{2}}{\epsilon_{\mathrm{w}}}=\delta \mathcal{G}_{\mathrm{e}}(x)-b u(x) \tau(x)
$$

with

$$
\tau(x)=-\frac{\sigma_{\mathrm{m}}^{2}}{\epsilon_{\mathrm{w}}} .
$$

Since the interface between the gold layer and the solution is much larger than the cantilever surface, one assumes that the modification of the cantilever surface does not induce a significant change in the overall charge density.

3.3.2. Displacement fields basis. To be able to represent the well-known localization effets on microelectrodes [33], one assumes that the cantilever is subjected to a heterogeneous shear-stress field

$$
\tau(x)=\frac{1}{\left(x_{0}-x\right)^{m}}
$$

where $x_{0}$ and $m$ are two parameters describing the stress field when $x<x_{0}$. According to the analysis of the system described in [24], the mechanical term $\phi_{\text {mec }}$ reads as

$$
\begin{aligned}
\phi_{\text {mec }}(x, y)= & \frac{4 \pi n}{\iota \lambda}(v(x, y)-v(x-d, y)) \\
& +\frac{\partial \phi_{\mathrm{W}}}{\partial \alpha}\left(\frac{\mathrm{d} v}{\mathrm{~d} x}(x, y)-\frac{\mathrm{d} v}{\mathrm{~d} x}(x-d, y)\right),
\end{aligned}
$$

where $v(x)$ is in the out-of-plane displacement field and $\partial \phi_{\mathrm{W}} / \partial \alpha$ is an experimentally identified coefficient, $n$ the average refractive index of the ambient medium, $\lambda$ the used wavelength and $\iota$ a scaling coefficient depending on the numerical aperture of the used objective lens [24]. The displacement and cross-section rotation fields are derived as a closed-form solution in appendix A, thus providing a description of the mechanical phase field $\phi_{\mathrm{mec}}$.

\section{Identification}

Section 3 provides a possible description of the measured phase field arising from the electroelastic coupling described in section 2. Figure 6 shows the measured optical phase recorded along the median line of the cantilever as a function of the loading step. This phase ranges (by definition) from $-\pi$ to $\pi$ and is thus subjected to phase jumps. No significant difference has been noted in the phase behaviour across the cantilever width, so that the following results are presented for the median (i.e. number 8) line of the cantilever, even though the identification procedures involve all the pixels covering the cantilever (i.e. 15-pixel rows). The aim of this section is to use the measured phase maps, taking explicitly into account both the electrochemical and the mechanical phase terms to propose a modelling of the electroelastic coupling. 


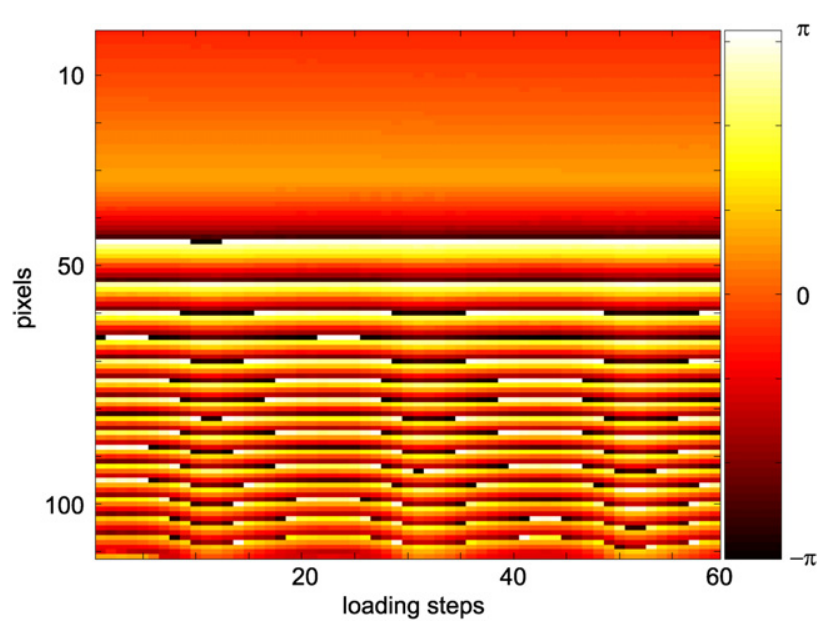

Figure 6. Measured phase recorded along the median line of the cantilever as a function of the loading step.

\subsection{Identification problem}

Solving a direct mechanical problem usually consists of finding stress and displacement fields satisfying simultaneously:

- kinematic compatibility,

- equilibrium conditions, and

- constitutive law.

A global formulation of the purely mechanical identification problem is to find the displacement field $\boldsymbol{U}$ minimizing $\varphi^{2}$

$$
\begin{aligned}
\varphi^{2}= & K_{\mathrm{KA}}\left\|\boldsymbol{U}-\boldsymbol{U}_{\mathrm{m}}\right\|_{\mathrm{KA}}+K_{\mathrm{SA}}\left\|\boldsymbol{U}-\boldsymbol{U}_{\mathrm{SA}}\right\|_{\mathrm{SA}} \\
& +K_{\mathrm{CE}}\left\|\boldsymbol{U}-\boldsymbol{U}_{\mathrm{CE}}\right\|_{\mathrm{CE}},
\end{aligned}
$$

that is matching 'at best' the measured displacement field (i.e. the kinematic conditions, first term), satisfying equilibrium conditions (second term) as well as the constitutive law (third term) [23]. As outlined by equations (6) and (31), the displacement field is not directly accessible, so that one has to modify the function $\varphi^{2}$ to use the optical phase instead of displacements and to take into account the ambivalent nature of the information carried by the measured optical phase:

- the phase decomposition reads $\phi_{\mathrm{mes}}=\phi_{\mathrm{mec}}+\phi_{\mathrm{ec}}$,

- the mechanical component $\phi_{\mathrm{mec}}$ represents the displacement field and thus appears in the first term (kinematic conditions) of equation (32),

- the electrochemical component $\phi_{\mathrm{ec}}$ is an image of the loading intensity on the cantilever and thus accounts for the second term (equilibrium conditions). It is obtained as $\phi_{\mathrm{ec}}(x, E)=h\left(\sigma_{\mathrm{m}}(x, E)\right)-h\left(\sigma_{\mathrm{m}}(x-d, E)\right)$.

Furthermore, contrary to most of the above-cited identification procedures where the last term in equation (32) involves only the (possibly heterogeneous) mechanical behaviour of the material under scrutiny, here one deals with coupled phenomena, thus involving an additional coupling term between the local electrochemical state and the local mechanical loading. For the sake of simplicity, one assumes in the following that the material mechanical behaviour is uniform along the cantilever. In order to avoid errors arising from phase jumps, one proposes to formulate the identification problem as finding a minimizer for

$$
\begin{aligned}
\eta^{2}= & \frac{1}{N_{p}} \sum_{x, y, n} \chi(x, y, n)^{2} \\
= & \frac{1}{N_{p}} \sum_{x, y, n} \frac{1}{\mu(x, y, n)^{2}}\left(\phi_{\text {test }}(x, y, n)-\phi_{\mathrm{test}}(x, y, n-1)\right. \\
& \left.-\phi_{\mathrm{mes}}(x, y, n)+\phi_{\mathrm{mes}}(x, y, n-1)\right)^{2}
\end{aligned}
$$

where $\phi_{\text {mes }}(x, y, n)-\phi_{\text {mes }}(x, y, n-1)$ is the measured phase change between two consecutive loading steps, $\phi_{\text {test }}(x, y, n)-$ $\phi_{\text {test }}(x, y, n-1)$ the phase change estimated from the actual parameters, $N_{p}$ the number of measurement points and $\mu(x, y, n)^{2}$ the estimated phase change variance at the considered point (see appendix B). One should underline that $\phi_{\text {test }}(x, y, n)$ depends on:

- the mechanical loading applied to the overall cantilever at step $n$ (through the $\phi_{\mathrm{mec}}$-term), and

- the mechanical loading applied to point $(x, y)$ and at step $n$, through the $\phi_{\mathrm{ec}}$-term.

Several assumptions have thus to be made to obtain an admissible phase field basis, that is a basis for phase fields satisfying simultaneously both equilibrium conditions and constitutive law(s). Minimizing the objective function defined by equation (33) yields then a projection of the measured phase map onto the defined basis, minimizing the first term of equation (32) (i.e. kinematic conditions). As the whole working electrode is gold coated (and not only the cantilever under scrutiny), the major difficulty to overcome is then to estimate the local charge-density field (on the cantilever) from global electrical measurements (i.e. on the whole electrode).

\subsection{Identification as a potential driven process}

Let us assume here that the global charge density of the cantilever may be estimated from the potential of the working electrode. As a consequence:

- the local charge density reads as $\sigma_{\mathrm{m}}(x, E)=$ $D(x) \times P(E)$,

- $D(x)=c_{p}\left(x_{0}\right) /\left(x_{0}-x\right)^{p}$ and the constant $c_{p}\left(x_{0}\right)$ is defined through the normalization condition $\int_{0}^{L} D(x) \mathrm{d} x=1$, by integrating the localization function over the cantilever length $L$.

- the local shear stress is a continuous function of the charge density $\tau=\delta \sigma_{\mathrm{m}}^{n}$,

- $P(E)$ is alternatively defined in two ways:

(a) $P(E)=\boldsymbol{B} C_{\mathrm{d}}\left(E-E_{\mathrm{PZC}}\right)$, where $C_{\mathrm{d}}$ is the (homogeneous) differential capacity of the surface, $\boldsymbol{B}$ is a multiplicative constant to be identified (both constant along the cycles) and $E_{\mathrm{PZC}}$ the zero-charge potential,

(b) $\mathrm{d} P(E)=B C_{\mathrm{da}} \mathrm{d} E$ during anodic scans, $\mathrm{d} P(E)=$ $\boldsymbol{B} C_{\mathrm{dc}} \mathrm{d} E$ during cathodic scans and $E_{\mathrm{PZC}}$ the zerocharge potential for the first scan. This last formulation allows one to account for a slight charge leak. 
Identification from full multi-physical fields

Table 1. Identified parameters for the different stress-charge relationships assuming a potential driven process.

\begin{tabular}{llllllllll}
\hline $\begin{array}{l}\text { Parameter } \\
\text { set }\end{array}$ & $p$ & $n$ & $x_{0} / L$ & $\begin{array}{l}E_{\mathrm{PZC}} \\
(\mathrm{V} / \mathrm{Ag}-\mathrm{AgCl})\end{array}$ & $\begin{array}{l}\delta \\
(\text { u.a. })\end{array}$ & $\begin{array}{l}\delta\left(\boldsymbol{B} C_{\mathrm{d}} c_{p}\left(x_{0}\right)\right)^{n} \\
(\text { a.u. })\end{array}$ & $\begin{array}{l}\theta_{\mathrm{e}} \\
(\mathrm{rad})\end{array}$ & $\begin{array}{l}C_{\mathrm{dc}} / C_{\mathrm{da}} \\
\eta_{\mathrm{min}}^{2}\end{array}$ \\
\hline $\mathrm{a}$ & 2.07 & 1.37 & 1.07 & -0.02 & $-2.47 \times 10^{-6}$ & -4.62 & 0.47 & 0.2 \\
$\mathrm{~b}$ & 1.45 & 1.71 & 1.12 & 0.06 & $-1.7 \times 10^{-7}$ & -13.73 & 0.16 & 0.77 & 0.19 \\
\hline
\end{tabular}

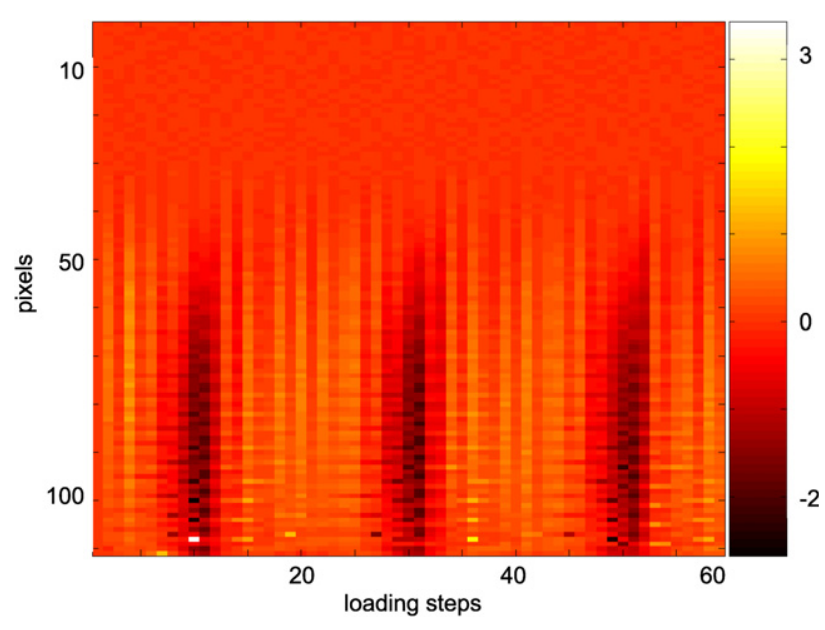

Figure 7. Residual map along the median line of the cantilever as a function of the loading step $\chi(x, 8, n)$ after minimization assuming a potential driven process and the b-set parameters.

Assuming that the cantilever edges are sharp would lead to impose $x_{0}=L$ and $p \leqslant 1$ to ensure that the the surface charge density is integrable along the cantilever. The charge density is then singular, which is no longer true if one takes into account a finite edge radius [33]. One then chooses to impose only that $x_{0}>L$, so that the saturation effect due to the edge radius is represented preserving the integrability condition.

The minimization of $\eta^{2}$ is then performed, to provide a parameters set suitable for describing the measured phase maps as well as a residual value $\eta_{\min }^{2}$. Even though the solution has been found to be dependent on the initial guess, the best solution sets (for both assumptions) are reported in table 1 . The charge-density field is found to be singular with $p$ around 2 . The stress-charge density is also found to be non-linear, with an $n$-exponent around 1.7 for the b-set. Moreover, using the b-set allows one to identify a differential capacity ratio $C_{\mathrm{dc}} / C_{\mathrm{da}}=0.77$, meaning that the electric potential is not the suitable state variable to describe the mechanical loading.

This last result is shown by considering the residual map along the median line of the cantilever as a function of the loading step in figure 7. This residual is dimensionless as it is the ratio of the phase gap over the expected phase deviation (see appendix B). The normalized residual is greater than 1 on the cantilever for almost every loading step, meaning that the residual arises from modelling errors instead of measurement uncertainties. The overall error indicator $\eta_{\min }^{2}$ is then about 0.2 , which is rather high compared with the fact that pixels on the substrate, for which the modelling error vanishes, are also involved. This is a good indication that this first modelling should be improved by enriching the description of the electroelastic coupling.
Table 2. Identified parameters for the different stress-charge expansions assuming a charge-density driven process.

\begin{tabular}{lllll}
\hline $\begin{array}{l}\text { Number of terms } \\
\text { in the coupling } \\
\text { relationship expansion }\end{array}$ & $p$ & $x_{0} / L$ & $\begin{array}{l}\theta_{\mathrm{e}} \\
(\mathrm{rad})\end{array}$ & $\begin{array}{l}\text { Residual } \\
\eta_{\min }^{2}\end{array}$ \\
\hline 1 & 3.41 & 1.13 & 0.58 & $6.33 \times 10^{-2}$ \\
2 & 3.41 & 1.13 & 0.60 & $6.33 \times 10^{-2}$ \\
3 & 3.35 & 1.12 & 0.43 & $6.27 \times 10^{-2}$ \\
4 & 3.32 & 1.12 & 0.51 & $6.27 \times 10^{-2}$ \\
5 & 3.31 & 1.12 & 0.60 & $6.27 \times 10^{-2}$ \\
\hline
\end{tabular}

\subsection{Identification as a charge-density driven process}

The enrichment is achieved by including the charge information $Q$ obtained from the electrochemical workstation by integrating the current going through the whole working electrode and assuming that $Q=0$ at the initial instant. The charge variation is obtained by numerically integrating all the voltammogram cycles, sampled with a $1 \mathrm{mV}$ resolution. The charge change is then down-sampled to the image acquisition frequency. The assumptions are the following.

- The local charge density reads as $\sigma_{\mathrm{m}}(x, Q)=D(x) \times Q$.

- The presence of the cantilevers on the electrode does not significantly modify the overall electrode charge compared with a standard planar electrode. As a consequence, one sets $D(x)=c_{q}\left(x_{0}\right) /\left(x_{0}-x\right)^{p}$ and the constant $c_{q}\left(x_{0}\right)$ is defined so that $D(x)=1$ when $x$ tends to the field border on the substrate. The function $D(x)$ is then a localization function, relating the local charge density to the uniform charge one would get on a standard planar electrode. This condition then just means that one assumes that this uniform electrode charge density is recovered 'far enough' from the cantilever, that is around $30 \mu \mathrm{m}$ from the cantilever base in the present case.

- The local shear-stress profile is a continuous function of the charge density, expanded onto a polynomial basis

$$
\tau=\sum_{t=1}^{T} \delta_{t} \sigma_{\mathrm{m}}^{t} .
$$

4.3.1. Identified charge-density field and stress-charge relationship. The minimization of $\eta^{2}$ is then performed for different values for $T$ ranging from 1 to 5 , to provide parameter sets representing the measured phase maps. After a standard minimization assuming a linear $(T=1)$ coupling relationship, the parameters are identified for greater $T$ values using a relaxation algorithm. This has been found to provide stable results with respect to the initial guess for the first minimization.

The solution sets are reported in table 2. The chargedensity field is found to be singular with $p$ around 3.3 and a charge-density singularity virtually located in front 

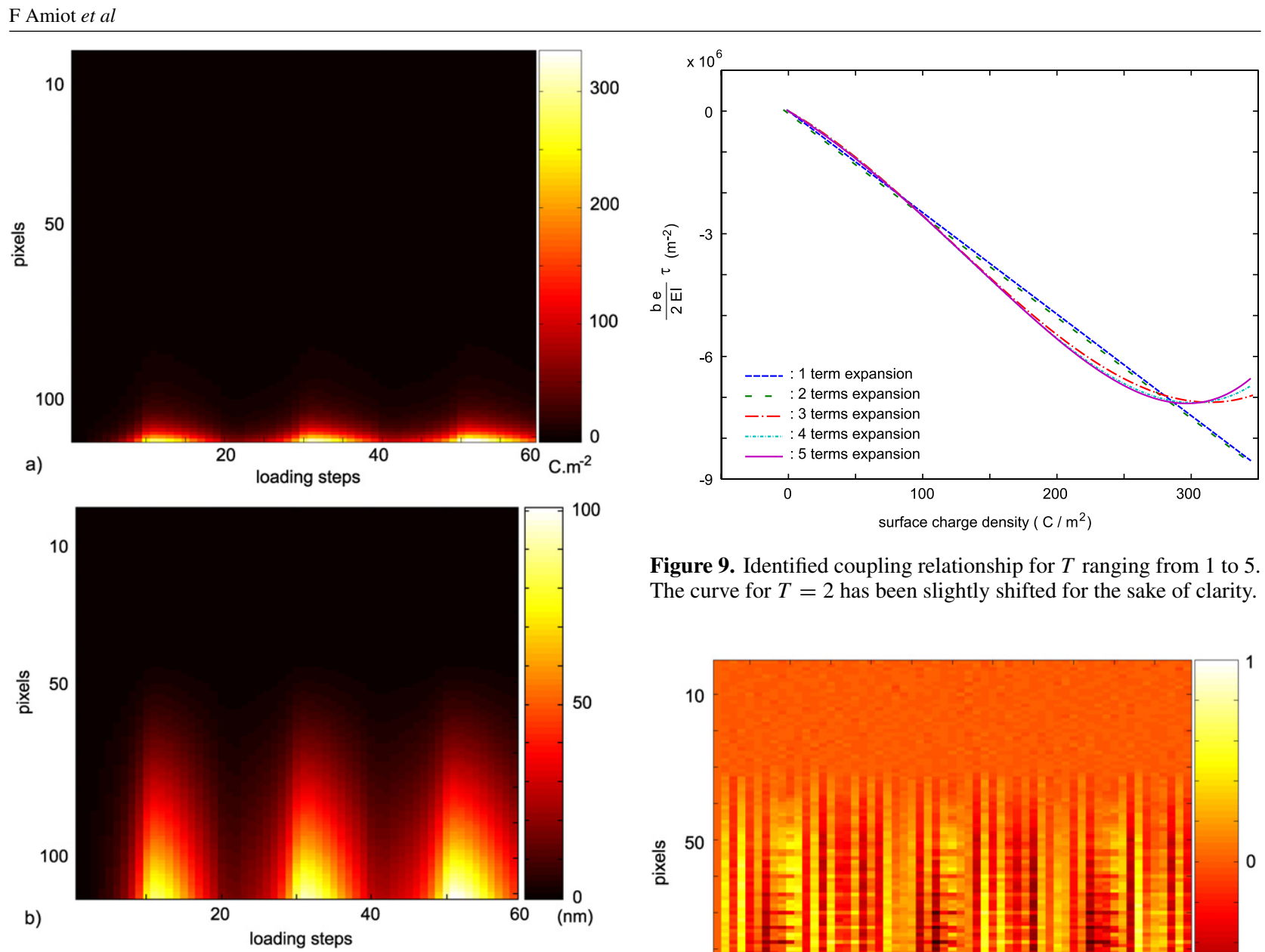

Figure 9. Identified coupling relationship for $T$ ranging from 1 to 5 . The curve for $T=2$ has been slightly shifted for the sake of clarity.

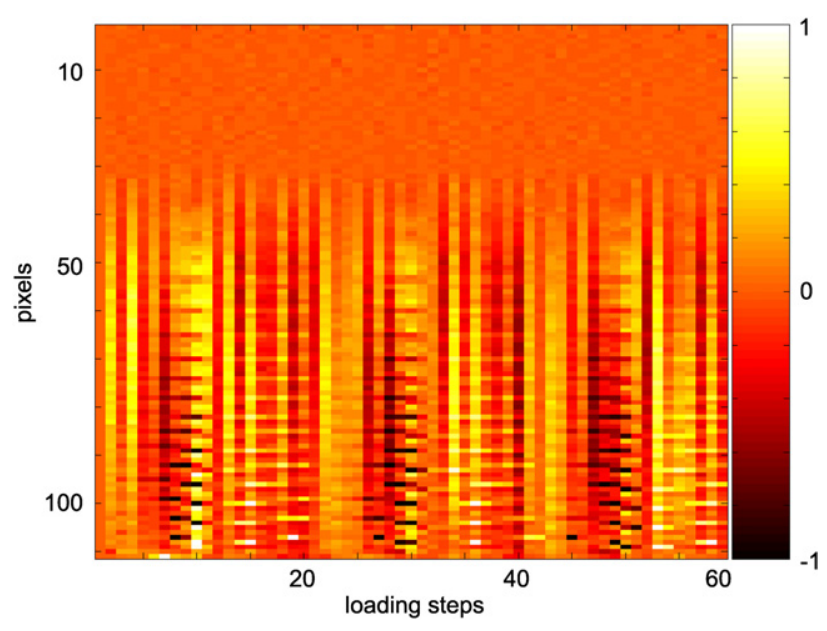

Figure 8. (a) Identified charge-density field along the median line of the cantilever as a function of the loading step. (b) Identified out-of-plane displacement field along the median line of the cantilever as a function of the loading step.

of the cantilever edge $\left(x_{0} / L=1.12\right)$, highlighting the microelectrode effect (see [34] for instance).

The resulting charge-density field along the median line of the cantilever is shown in figure $8(a)$. The ratio of the maximum charge density to its average is around 2000, outlining the high charge localization on microelectrodes. It is worth noting that even if $p>1$, the charge density is integrable since $x_{0}>L$. The induced mechanical effect yields a maximum upward displacement of about $100 \mathrm{~nm}$ at the end of the cantilever, as shown in figure $8(b)$. This corresponds to a measured phase change mainly arising from the mechanical term, except at the cantilever edge, where the electrochemical term is dominant. Even with a 'crude' linear coupling relationship, the overall residual $\eta_{\min }^{2}$ is down to $6.33 \times 10^{-2}$. It is worth noting that this is achieved using only 4 parameters compared with the 8 parameters of the b-set defined in section 4.2.

The overall residual slightly decreases by using more terms in the coupling expansion. The resulting coupling relations are shown in figure 9. By increasing the number of terms in the coupling relationship expansion, one reveals an inflexion point reached for a charge density of about $250 \mathrm{C} \mathrm{m}^{-2}$, with a stress saturation for $\sigma_{\mathrm{m}}>\sigma_{\mathrm{s}}=250 \mathrm{C} \mathrm{m}^{-2}$, whereas the low charge-density part is concave. One should

Figure 10. Residual map along the median line of the cantilever as a function of the loading step $\chi(x, 8, n)$ after minimization assuming a charge-density driven process and a 5-term expansion for the stress-charge-density relationship.

outline that this qualitatively agrees with the description derived in section 3.3.1, even though the identified exponent for charge densities less than $200 \mathrm{C} \mathrm{m}^{-2}$ is 1.09 to be compared with the 2-exponent deduced in section 3.3.1. It should be emphasized that the latter strongly depends on the kinematic model used to describe the cantilever behaviour (see equation (26)). It is also worth noting that the existence of an inflexion point in the coupling relation is consistent with a specific anion adsorption, which makes the surface expansion more favourable and which has been found to occur in an almost reversible manner for $\mathrm{Au}\left(\begin{array}{lll}1 & 1 & 1\end{array}\right)-\mathrm{Cl}^{-}$systems [35-37]. Last, it should be noted that these results are not significantly modified by any 'regularization' attempt to cancel the Ti underlayer passivation contribution to the overall electrode charge.

4.3.2. Residual map. Figure 10 shows the residual map along the median line of the cantilever as a function of the loading step $\chi(x, 8, n)$ for a 5-terms expansion of the 
coupling relationship. The normalized phase error is found to remain almost everywhere in the $[-1,1]$ range, meaning this mainly arises from measurement uncertainties. It should be underlined that the decrease in $\eta^{2}$ when revealing the inflexion point (see table 2) is small compared with the overall value because the modification only concerns a few loading steps, where the surface charge density reaches $\sigma_{\mathrm{s}}$. Furthermore, the error pattern close to the loading steps where the electrode potential is $0.4 \mathrm{~V}$ (that is steps 10,30 and 50 in figure 10) exhibits negative error values during anodic scans and positive values during cathodic scans. This symmetric shape, which is preserved even though the images obtained for an electrode potential of $0.4 \mathrm{~V}$ (where the current varies very suddenly) are removed, tends to show that further improvements in the mechanical modelling of the observed effects may be achieved when considering partly inelastic stresses.

\section{Conclusion}

Metal coated microcantilevers were utilized as microelectrodes. Full-field interferometric measurements were carried out to monitor both the surface composition changes and the cantilever displacement during cyclic voltammetry in the double-layer region. The scaling of the electrochemically induced phase with respect to the surface charge density is modelled according to Gouy-Chapman-Stern theory, whereas the relationship between the mechanical effect and the surface charge density is identified thanks to a dedicated formulation of the identification problem. The electroelastic coupling effect is found to be charge-driven. The charge-density field is also found to be singular on the cantilever edge, and the shear stress versus charge density is shown to be non-linear, exhibiting an inflexion point that may be the signature of specific anion adsorption.

\section{Appendix A. Displacement fields basis}

One assumes a priori that the cantilever is subjected to a heterogeneous shear-stress field

$$
\tau(x)=\frac{1}{\left(x_{0}-x\right)^{m}},
$$

where $x_{0}$ and $m$ are two parameters describing the stress field when $x<x_{0}$. The bending moment $M_{f}(x)$ reads as

$$
\begin{aligned}
M_{f}(x) & =-\frac{b e_{1}}{2} \int_{x}^{L} \frac{\mathrm{d} \eta}{\left(x_{0}-\eta\right)^{m}} \\
& =-\frac{b e_{1}}{2} \ln \left(\frac{x_{0}-x}{x_{0}-L}\right) \quad \text { if } m=1 \\
& =-\frac{b e_{1}}{2(m-1)}\left(\left(x_{0}-L\right)^{1-m}-\left(x_{0}-x\right)^{1-m}\right) \\
& \quad \text { if } m \neq 1,
\end{aligned}
$$

where $L$ is the cantilever's length. By assuming that the bending stiffness is uniform all along the beam, the rotation and displacement fields are obtained assuming that the beam is described by an Euler-Bernoulli model [38] with a constant bending stiffness $E_{\mathrm{B}} I_{\mathrm{B}}$, where $E_{b}$ and $I_{b}$ are the average Young's modulus and quadratic moment, respectively. The cross-section rotation field is obtained as a function of the exponent $m$ by setting $\mathrm{d} v(0) / \mathrm{d} x=0$ :

$$
\begin{aligned}
\frac{\mathrm{d} v(x)}{\mathrm{d} x}= & -\frac{b e_{1}}{2 E_{b} I_{b}}\left\{\left(x-x_{0}\right) \ln \left(\frac{x-x_{0}}{L-x_{0}}\right)\right. \\
& \left.-x+x_{0} \ln \left(\frac{x_{0}}{x_{0}-L}\right)\right\} \quad \text { if } m=1 \\
= & -\frac{b e_{1}}{2 E_{b} I_{b}}\left\{\ln \left(\frac{x_{0}-x}{x_{0}}\right)+\frac{x}{\left(x_{0}-L\right)}\right\} \quad \text { if } m=2 \\
= & -\frac{b e_{1}}{4 E_{b} I_{b}}\left\{-\frac{1}{x_{0}-x}+\frac{x}{\left(x_{0}-L\right)^{2}}+\frac{1}{x_{0}}\right\} \text { if } m=3 \\
= & -\frac{b e_{1}}{2 E_{b} I_{b}(m-1)}\left\{\frac{\left(x_{0}-x\right)^{-m+2}-x_{0}^{-m+2}}{-m+2}\right. \\
& \left.+x\left(x_{0}-L\right)^{-m+1}\right\} \quad \text { if } m \neq\{1,2,3\}
\end{aligned}
$$

and the displacement field is obtained by setting $v(0)=0$

$$
\begin{aligned}
v(x)= & \frac{b e_{1}}{4 E_{b} I_{b}}\left\{-\left(x-x_{0}\right)^{2} \ln \left(\frac{x-x_{0}}{L-x_{0}}\right)\right. \\
& \left.-x_{0}\left(2 x-x_{0}\right) \ln \left(\frac{x_{0}}{x_{0}-L}\right)-x\left(x_{0}-\frac{3 x}{2}\right)\right\} \\
= & -\frac{b e_{1}}{2 E_{b} I_{b}}\left\{\left(x-x_{0}\right) \ln \left(\frac{x_{0}-x}{x_{0}}\right)\right. \\
& \left.-x\left(1-\frac{x}{2\left(x_{0}-L\right)}\right)\right\} \quad \text { if } m=2 \\
= & -\frac{b e_{1}}{4 E_{b} I_{b}}\left\{\ln \left(\frac{x_{0}-x}{x_{0}}\right)+\frac{1}{2}\left(\frac{x}{x_{0}-L}\right)^{2}+\frac{x}{x_{0}}\right\} \\
= & -\frac{\text { if } m=3}{2 E_{b} I_{b}(m-1)}\left\{\frac{x_{0}^{-m+3}-\left(x_{0}-x\right)^{-m+3}}{(-m+2)(-m+3)}\right. \\
& \left.+\frac{x^{2}}{2}\left(x_{0}-L\right)^{-m+1}-x \frac{x_{0}^{-m+2}}{-m+2}\right\} \text { if } m \neq\{1,2,3\}
\end{aligned}
$$

\section{Appendix B. Estimation of the measured phase variance}

It can be shown [24] that using four integrating buckets and a sinusoidal phase modulation yields four intensity images $E_{p}$, for $p=\{1,2,3,4\}$,

$$
\begin{aligned}
E_{p}= & \frac{T}{4}\left(I_{0}+A J_{0}(\psi) \cos (\phi)\right)+\frac{T A \cos (\phi)}{\pi} \sum_{n=1}^{\infty} \frac{J_{2 n}\left(\psi_{0}\right)}{2 n} \\
& \times\left[\sin \left(n p \pi+2 n \theta_{\text {mod }}\right)-\sin \left(n(p-1) \pi+2 n \theta_{\text {mod }}\right)\right] \\
& -\frac{T A \sin (\phi)}{\pi} \sum_{n=0}^{\infty} \frac{J_{2 n+1}\left(\psi_{0}\right)}{2 n+1} \\
& \times\left(\cos \left(\frac{\pi}{2}(2 n+1)(p-1)+(2 n+1) \theta_{\text {mod }}\right)\right. \\
& \left.-\cos \left(\frac{\pi}{2}(2 n+1) p+(2 n+1) \theta_{\text {mod }}\right)\right)
\end{aligned}
$$


where $J_{n}$ is the first kind Bessel function of $n$th order. The images $E_{p}$ depend on average impinging intensity $I_{0}$ and on $\cos (\phi)$ and $\sin (\phi)$ where $\phi$ is the optical phase to be measured. Using four independent images then provides enough information to recover three parameters, including $\phi$. For each pixel of the CCD array, the ideal set of equation (B.1) is rewritten as a linear system of equations:

$$
\mathcal{M P}=\boldsymbol{E},
$$

where the parameters vector $\boldsymbol{P}$ reads as

$$
\boldsymbol{P}^{t}=\left[\frac{T I_{0}}{4}, \frac{T A}{\pi} \cos (\phi), \frac{T A}{\pi} \sin (\phi)\right]
$$

and the images vector

$$
\boldsymbol{E}^{t}=\left[E_{1}, E_{2}, E_{3}, E_{4}\right]
$$

with $(\cdot)^{t}$ the transpose of $(\cdot)$. The matrix $\mathcal{M}$ is built from the modulation parameters and is then independent of the considered point. Let us denote by $\boldsymbol{\lambda}_{\mathrm{m}}$ the given (measured) realization of a Poisson process whose intensity is $E_{p}$. The solution parameter vector $\boldsymbol{P}_{\text {sol }}$ is obtained as the likelihood maximizer, that is as the minimizer of

$L(\boldsymbol{P})=\sum_{i=1}^{4}\left\{\ln \left(\Gamma\left(\lambda_{m, i}\right)\right)-\ln \left(\Gamma\left(\lambda_{i}\right)-\left(\lambda_{m, i}-\lambda_{i}\right) \ln \left(\lambda_{i}\right)\right)\right\}$

with respect to the parameter set $\boldsymbol{P}$

$$
\lambda=\mathcal{M P},
$$

where $\boldsymbol{\lambda}$ denotes the actual intensity set. The stationarity conditions read

$$
g\left(\boldsymbol{\lambda}_{\mathrm{m}}, \mathbf{P}\right)=\mathcal{M}^{t} f(\boldsymbol{\lambda})-\mathcal{M}^{t} \boldsymbol{r}=0,
$$

where

$$
\begin{aligned}
& f\left(\lambda_{i}\right)=\ln \left(\lambda_{i}\right)-\Psi\left(\lambda_{i}\right)+1, \\
& r_{i}=\frac{\lambda_{m, i}}{\lambda_{i}},
\end{aligned}
$$

and $\Gamma(x)$ is the Gamma-Euler function and $\Psi(x)$ the derivative of $\ln (\Gamma(x))$ with respect to $x$. Considering small perturbations from a solution $\left(\boldsymbol{\lambda}_{\mathrm{s}}=\boldsymbol{E}, \boldsymbol{P}_{\mathrm{s}}\right)$, a Taylor expansion of $g$ yields

$$
\mathcal{M}^{t} \mathcal{N} \mathcal{M}\left(\boldsymbol{P}-\boldsymbol{P}_{\mathrm{s}}\right)=\mathcal{M}^{t} \boldsymbol{R}
$$

with

$$
\begin{aligned}
& N_{i, j}=0 \quad \text { if } i \neq j \\
& N_{i, i}=\left(\frac{\mathrm{d} f}{\mathrm{~d} \lambda}\left(\lambda=\lambda_{s, i}\right)+\frac{1}{\lambda_{s, i}}\right) \\
& R_{i}=\frac{\lambda_{i}-\lambda_{s, i}}{\lambda_{s, i}},
\end{aligned}
$$

so that the error on the parameters $\boldsymbol{P}$ is a linear combination of the normalized error on the measured intensities $\boldsymbol{R}$

$$
\delta \boldsymbol{P}=\boldsymbol{P}-\boldsymbol{P}_{\mathrm{s}}=\mathcal{A} \boldsymbol{R},
$$

where

$$
\mathcal{A}=\left(\mathcal{M}^{t} \mathcal{N} \mathcal{M}\right)^{-1} \mathcal{M}^{t}
$$

The couple $\left(P_{2}, P_{3}\right)=\left(T A \cos \left(\phi_{\mathrm{sol}}\right) / \pi, T A \sin \left(\phi_{\mathrm{sol}}\right) / \pi\right)$ is extracted from the parameter vector $\boldsymbol{P}$ and used as the argument of a standard 'atan2' function to provide a less corrupted value of the phase. As a consequence, errors $\delta \boldsymbol{P}$ on the identified $\boldsymbol{P}$ vector induce a phase bias $\delta \phi$ that relates to $\delta \mathbf{P}$ as

$$
\tan (\delta \phi)^{2} \simeq \frac{\left(\delta P_{3} \cos (\phi)-\delta P_{2} \sin (\phi)\right)^{2}}{\left(P_{2}^{2}+P_{3}^{2}\right)}
$$

A (conservative) estimator $\epsilon$ for $\delta \phi$ is then provided by

$$
\epsilon=\tan ^{-1}\left(\sqrt{\int_{0}^{2 \pi} \tan (\delta \phi(\phi))^{2} \mathrm{~d} \phi}\right) .
$$

The quantity $\epsilon$ depends on $\left(\delta P_{2}\right)^{2}$ and $\left(\delta P_{3}\right)^{2}$, which are estimated by

$$
\left(\delta P_{k}\right)^{2}=\sum_{l=1}^{4} \mathcal{A}_{k, l}^{2} \frac{1}{\lambda_{l}},
$$

so that $\epsilon$ depends on the considered point $(x, y, n)$ in the phase maps, and the variance $\mu(x, y, n)^{2}$ of the difference between two consecutive phase measurements is estimated as

$$
\mu(x, y, n)^{2}=\epsilon(x, y, n)^{2}+\epsilon(x, y, n-1)^{2} .
$$

\section{References}

[1] Lavrik N V, Sepaniak M J and Datskos P G 2004 Rev. Sci. Instrum. 75 2229-53

[2] Marichev V A 2005 Surf. Sci. Rep. 56 277-324

[3] Jaeckel L, Lang G and Heusler K E 1994 Electrochim. Acta 39 1031-8

[4] Raiteri R and Butt H J 1995 J. Phys. Chem. 99 15728-32

[5] Ibach H, Bach C E, Giesen M and Grossman A 1997 Surf. Sci. 375 107-19

[6] Tian F, Pei J H, Hedden D L, Brown G M and Thundat T 2004 Ultramicroscopy 100 217-23

[7] Tabard-Cossa V, Godin M, Beaulieu L Y and Grütter P 2005 Sensors Actuators B 107 233-41

[8] Brunt T A, Rayment T, O'Shea S J and Welland M E 1996 Langmuir 12 5942-6

[9] Stedman M 1968 Chem. Phys. Lett. 2 457-60

[10] Stedman M 1970 Symp. on Optical Studies of Absorbed Layers at Interfaces (London: Faraday Society) pp 64-71

[11] Paik W-P, Genshaw M A and Bockris J O M 1970 J. Phys. Chem. 74 4266-75

[12] Godin M, Laroche O, Tabard-Cossa V, Beaulieu L Y, Grütter P and Williams P J 2003 Rev. Sci. Instrum. 74 4902-7

[13] Kohn R V and Lowe B D 1988 Math. Mod. Numer. Anal. 22 119-58

[14] Ladevèze P 1975 Comparaison de modèles de milieux continus Thèse d'Etat Université Paris 6

[15] Mottershead J E and Friswell M I 1993 J. Sound Vib. $167347-75$

[16] Geymonat G, Hild F and Pagano S 2002 C. R. Mec. 330 403-8

[17] Calloch S, Dureisseix D and Hild F 2002 Technol. Form. 100 36-41

[18] Grédiac M 1989 C. R. Acad Sci. Paris 309 1-5

[19] Grédiac M, Toussaint E and Pierron F 2002 Int. J. Solids Struct. 39 2691-705

[20] Bui H D 1995 2e Colloque National de Calcul Des Structures (Paris: Hermes) pp 25-35 
[21] Bonnet M and Constantinescu A 2005 Inverse Probl. 21 1-50

[22] Claire D, Hild F and Roux S 2002 C. R. Mec. 330 729-34 See also: Claire D, Hild F and Roux S 2004 Int. J. Numer. Methods Eng. 61 189-208

[23] Amiot F, Hild F and Roger J P 2006 Int. J. Solids Struct. 44 2863-87

[24] Amiot F and Roger J P 2006 Appl. Opt. 45 7800-10

[25] Gouy G 1910 J. Physique 9 457-68

[26] Chapman D L 1913 Phil. Mag. 25 475-81

[27] Andelman D 2005 Nato ASI and SUSSP Conf. on Soft Condensed Matter Physics in Molecular and Cell Biology (Edinburgh, UK)

[28] Zana R and Yeager E 1967 J. Phys. Chem. 71 521-36

[29] Waddington T C 1966 Trans. Faraday Soc. 621482

[30] Born M and Wolf E 1980 Principles of Optics (Oxford: Pergamon)
[31] Shatalin S V, Tan J B, Juskaitis R and Wilson T 1995 Opt Commun. 116 291-9

[32] Dubois A 2004 Appl. Opt. 43 1503-7

[33] Van Bladel J 1996 Singular Electromagnetic Fields and Sources (Oxford: Wiley-IEEE)

[34] Maus R G, McDonald E M and Wightman R M 1999 Anal. Chem. 71 4944-50

[35] Clavilier J and Van Huong N 1973 Electroanal. Chem. Interfacial Electrochem. 41 193-9

[36] Van Huong N, Clavilier J and Bonnemay M 1975 J. Electroanal. Chem. 65 531-43

[37] Lei H-W, Uchida H and Watanabe M 1997 Langmuir 13 3523-8

[38] Timoshenko S P and Goodier J N 1970 Theory of Elasticity 3rd edn (New York: McGraw-Hill) 